\title{
On the influence of a network on optically isotropic fluid phases with tetrahedral/octupolar order
}

\author{
Helmut R. Brand ${ }^{1,2, a}$ and Harald Pleiner ${ }^{2, b}$ \\ 1 Theoretische Physik III, Universität Bayreuth, 95440 Bayreuth, Germany \\ 2 Max-Planck-Institute for Polymer Research, POBox 3148, 55021 Mainz, Germany
}

Received 20 October 2016

Published online: 27 March 2017

(C) The Author(s) 2017. This article is published with open access at Springerlink.com

\begin{abstract}
We investigate the influence of transient or permanent elasticity on liquid phases with octupolar (tetrahedral) order, a question that has never been addressed before. The focus will be on optically isotropic liquid phases with tetrahedral order including the $\mathrm{T}_{d}$ phase and the chiral $\mathrm{T}$ phase introduced by Fel. It turns out that the presence of both, a network as well as tetrahedral order can lead to the formation of chiral domains of both hands in an optically isotropic fluid due to a completely novel mechanism, thus providing a possible macroscopic explanation for recent experimental observations. We study in detail how elasticity influences the macroscopic dynamics of both, the $\mathrm{T}_{d}$ and the $\mathrm{T}$ phase. The simultaneous presence of a transient network as well as of octupolar order is shown to lead to completely new crosscoupling phenomena for optically isotropic systems including transient dissipative elastic strains due to temperature gradients.
\end{abstract}

\section{Introduction}

An open question in the field of condensed matter physics as well as in biophysics is the issue of mesoscopic and macroscopic mechanisms for chiral symmetry breaking in optically isotropic condensed systems.

Over the last decade or so it has become clear in the field of liquid crystals formed by bent-core (bananashaped) molecules that mesoscopic and macroscopic chiral domains of opposite hand can arise spontaneously in compounds composed of achiral molecules [1-5]. This phenomenon has been modeled by the simultaneous presence of octupolar/tetrahedral order as well as of conventional quadrupolar orientational order [6-8] (compare ref. [9] for a recent review of the general impact of octupolar order in the field of liquid crystalline phases).

It turns out that the observed chiral domains of both hands in compounds composed of achiral molecules can be either due to ambidextrous chirality based on a structural origin or due to ambidextrous helicity which is exclusively based on the existence of a linear gradient term which can generate helices of either hand [6-9]. The phenomenon of ambidextrous chirality and ambidextrous helicity is thus clearly distinguishable from the frequently observed helix formation in cholesteric and chiral smectic

\footnotetext{
a e-mail: brand@uni-bayreuth.de

b e-mail: pleiner@mpip-mainz.mpg.de
}

liquid crystals [10-12], which is due to molecular chirality whose effects are transported to a macroscopic scale by the collective effect of forming helical superstructures.

Quite recently isotropic-isotropic phase transitions have been observed for several compounds composed of achiral molecules with liquid crystalline phases following at lower temperatures [13-17]. In one case a liquid crystalline tetragonal phase showing a linear electro-optic effect was observed at lower temperatures [13], while in the other three cases Tschierske's group reported the existence of mesoscopic to macroscopic chiral domains of either hand in the lower temperature optically isotropic phase [14-16]. This observation was linked to the formation of clusters and or agglomerates of substantial size and associated with steric and packing interactions [14-16]. It is worth noticing that all the molecules forming these clusters and/or agglomerates are fairly large and bulky and rather distinct from typical rod-like molecules forming usual low molecular weight nematic phases.

In the present paper we will focus completely on optically isotropic fluid phases, which are characterized by the simultaneous presence of optically isotropic octupolar order and of a transient network. In this context we model the clusters and/or agglomerates as a transient network and investigate its influence on an optically isotropic phase, which has actually octupolar order (the $\mathrm{T}_{d}$ phase in the notation of ref. [18]). It turns out that in such a (visco)elastic $\mathrm{T}_{d}$ phase, which we will call $\varepsilon \mathrm{T}_{d}$ phase in 
the following, a linear gradient term coupling octupolar order and elasticity is possible. We show that this crosscoupling term not considered before leads to ambidextrous helicity and thus provides a possible explanation for the chiral domains of both hands observed in refs. [14-16]. Or to phrase it more succinctly: we suggest a novel scenario of chiral symmetry breaking in the mesoscopic and macroscopic domain, which might be useful also for other areas from condensed matter and biological physics.

To further elucidate our suggestions we derive the complete macroscopic dynamics of the optically isotropic $\varepsilon \mathrm{T}_{d}$ phase, by taking into account both, the hydrodynamic degrees of freedom related to octupolar order, and a strain tensor related to a network. In the case of a transient network the strain is relaxing, while for permanent networks the strain is diffusing. In addition we provide an appropriate discussion for an optically isotropic chiral T phase [18] that is modified by the presence of elasticity, which we will call $\varepsilon \mathrm{T}$ phase in the following.

We emphasize that the conventional quadrupolar nematic order parameter $Q_{i j}$ [10] plays absolutely no role throughout the present paper. Therefore the twist-bend nematic phase, which has been of great interest recently (compare, for example, sect. 4.4 of the recent review by Tschierske and Ungar [17]), and which we consider to be an interesting challenge for the macroscopic modeler, is not a subject of the present paper. We mention in passing that quite frequently twist-bend nematic phases show a nematic to nematic phase transition where typically one of the nematic phases involved is thought to be a conventional nematic phase with quadrupolar orientational order [19-21]. Finally, we mention ref. [22] as an example of a paper discussing the simultaneous presence of octupolar and quadrupolar nematic order from a microscopic point of view. Indeed, this work, which has no transient strain, but conventional nematic order, has been discussed in our recent review (ref. [9]) on phases with the simultaneous presence of octupolar and conventional quadrupolar nematic order.

The present paper is organized as follows. In sect. 2 we describe Ginzburg-Landau considerations for the $\varepsilon \mathrm{T}_{d}$ phase as well as for the $\varepsilon \mathrm{T}$ phase. In sect. 3 we derive in detail the resulting dynamic macroscopic equations for both phases. In this analysis static aspects of permanent elasticity are naturally included as well. In sect. 4 we investigate some simple solutions of the macroscopic equations derived that can be tested experimentally. Finally, we present in sect. 5 a brief summary and conclusions. In the appendix we discuss the simplifications of the dynamics for the special case that the network is permanent rather than transient.

\section{Ginzburg-Landau considerations}

\subsection{The elastic $\varepsilon T_{d}$ phase}

To characterize the octupolar order we make use of the order parameter $T_{i j k}[18]$ :

$$
T \equiv T_{i j k}=T_{0} \Sigma_{\alpha=1}^{4} n_{i}^{\alpha} n_{j}^{\alpha} n_{k}^{\alpha}
$$

expressed by the 4 unit vectors, $\boldsymbol{n}^{\alpha},(\alpha=1, \ldots, 4)$ spanning a tetrahedron and with $T_{0}$ the strength of the octupolar order. The two possibilities of orientation of the tetrahedron are taken into account assigning a positive and a negative sign to $T_{0}$ reflecting the broken inversion symmetry. Since the sum of the 4 tetrahedron vectors vanishes, $T_{i j j} \equiv 0$ guaranteeing the absence of polar order. Using for $T i j k$ the normalization $T_{i k l} T_{j k l}=\alpha \delta_{i j}$, we find $\alpha=(32 / 27) T_{0}^{2}[7]$.

The Ginzburg-Landau analysis for the vicinity of the isotropic- $\mathrm{T}_{d}$ phase transition has been given previously in several papers [18,23-25], where also the absence of a cubic term in a Landau expansion in the order parameter $T_{i j k}$ has been elucidated [18,23-25]. When it comes to the coupling to electric fields, Fel [18] pointed out that a term $\sim T_{i j k} E_{i} E_{j} E_{k}$ enters a Landau expansion. While a cubic term in a magnetic field cannot exist due to a violation of time reversal symmetry $[9,26]$, a term quadratic in magnetic fields and linear in an electric field is possible $[9,26]$

$$
\varepsilon^{E H}=-\frac{\varepsilon_{2}}{3} T_{i j k}\left[E_{i} H_{j} H_{k}+E_{j} H_{i} H_{k}+E_{k} H_{j} H_{k}\right] .
$$

The Ginzburg term for $T_{i j k}$ takes the form

$$
\varepsilon^{G}=\delta_{1}\left(\nabla_{k} T_{i j l}\right)^{2}+\delta_{2}\left(\nabla_{l} T_{l j k}\right)\left(\nabla_{m} T_{m j k}\right) .
$$

Here we consider for the first time the influence of a transient and/or permanent strain field on phase transitions involving octupolar/tetrahedratic phases in the Ginzburg-Landau approximation. For the strain field $\varepsilon_{i j}$ associated with a network we find in harmonic approximation including also a gradient term in the spirit of Ginzburg-Landau

$$
\begin{aligned}
\varepsilon^{e l}= & c_{i j k l} \varepsilon_{i j} \varepsilon_{k l} \\
& +\gamma_{1}\left(\nabla_{k} \varepsilon_{i i}\right)\left(\nabla_{k} \varepsilon_{j j}\right)+\gamma_{2}\left(\nabla_{k} \varepsilon_{i i}\right)\left(\nabla_{l} \varepsilon_{k l}\right) \\
& +\gamma_{3}\left(\nabla_{k} \varepsilon_{i j}\right)\left(\nabla_{k} \varepsilon_{i j}\right)+\gamma_{4}\left(\nabla_{i} \varepsilon_{i k}\right)\left(\nabla_{j} \varepsilon_{j k}\right) .
\end{aligned}
$$

It turns out that the Ginzburg term for the strain tensor has thus four independent coefficients in the isotropic phase. We note that this result contains the structure of the Ginzburg term for the usual isotropic-nematic transition [27], since in the latter case the analogues of $\gamma_{1}$ and $\gamma_{2}$ involving $Q_{i j}$ vanish identically due to the vanishing trace $Q_{i i} \equiv 0$. The elastic tensor $c_{i j k l}$ takes its standard isotropic form [28]. obtain

For the coupling terms linear in the strain field $\varepsilon_{i j}$ we

$$
\varepsilon^{e l c}=\zeta\left(\varepsilon_{i j} E_{k}+\varepsilon_{i k} E_{j}+\varepsilon_{j k} E_{i}\right) T_{i j k}+\xi T_{i j k} \nabla_{k} \varepsilon_{i j} .
$$

We note that the contribution $\sim \zeta$ corresponds to piezoelectricity and has been given in the context of tetrahedral phases very recently in a review on octupolar order in liquid crystals [9]. An experimental candidate for which such a piezoelectric coupling plays a role is ref. [13], where it was found that a tetragonal liquid crystalline phase (below two optically isotropic phases at higher temperatures) shows a linear response to an applied electric field. A natural explanation for the behavior observed would be an 
actual phase sequence isotropic liquid - $\mathrm{T}_{d}$ phase - tetragonal phase with octupolar order.

Of particular interest is the linear gradient term given in eq. (5) representing a coupling of tetrahedral order to gradients of the strain field. A coupling of this structure can lead to helices of either hand (ambidextrous helicity) in a nonchiral system, as has been shown for an analogous linear gradient coupling between the nematic order parameter (director) and tetrahedral order [6-9]. It thus leads to a spontaneous mirror symmetry breaking in an optically isotropic liquid phase of achiral molecules. Such a spontaneous mirror symmetry breaking has indeed been observed very recently by the group of Tschierske [14-16]. In two cases $[14,16]$ the chiral domains appeared in an optically isotropic liquid phase below a truly isotropic liquid phase, while in the other case [15] chiral domains of opposite hand were arising as a reentrant optically isotropic liquid phase below a truly isotropic liquid phase and a nematic phase.

Starting from the relevant part of the energy

$$
F=F_{0}+\xi T_{i j k} \nabla_{k} \varepsilon_{i j}+\gamma\left(\nabla_{k} \varepsilon_{i j}\right)^{2}+\delta\left(\nabla_{k} T_{i j l}\right)^{2},
$$

where $F_{0}$ contains the spatially homogeneous terms in $T_{i j k}$ and $\varepsilon_{i j}$. In addition, we have used a simplified version of the Ginzburg terms for $\varepsilon_{i j}$ and $T_{i j k}$. Minimization with respect to the wave vector $q$ for a sinusoidal modulation of $\varepsilon_{i j}$ and $T_{i j k}$ yields

$$
q_{c}=\frac{2 \xi}{3 \sqrt{3}} \frac{1}{\gamma+64 \delta / 27}
$$

and an associated energy reduction

$$
\Delta f=-\frac{8 \xi^{2}}{27 \gamma+64 \delta}
$$

independent of the sign of $\xi$ for co-rotating helices of $T_{i j k}$ and $\varepsilon_{i j}$.

This analysis for an optically isotropic fluid system presents a mechanism never considered before to explain the spontaneous occurence of mesoscopic or macroscopic chiral domains of either hand in the framework of a continuum description.

\subsection{The elastic chiral $\varepsilon T$ phase}

In a tetrahedral phase made of chiral molecules, the $\mathrm{T}$ phase, the existence of a pseudoscalar quantity $q_{0}$ allows for additional chiral terms in a Ginzburg-Landau expansion. For the elastic chiral $\varepsilon \mathrm{T}$ phase they read

$$
\begin{aligned}
F^{\text {chir }}= & q_{0} K^{T} \epsilon_{i j k} T_{i l m} \nabla_{j} T_{k l m} \\
& +q_{0} \xi^{e T} \epsilon_{i j k}\left(\nabla_{m} \varepsilon_{l j}\right)\left(\nabla_{i} T_{k l m}\right) .
\end{aligned}
$$

The first term in eq. (9) is of the same structure as known from the cholesteric-isotropic phase transition when $T_{i j k}$ is replaced by $Q_{i j}$. Apparently it has not been considered before. The second term in eq. (9) is a higher-order gradient term when compared to the linear gradient term applicable to achiral and chiral systems, which has been given above in eq. (5).

\section{Macroscopic dynamics}

In this section we present the full set of macroscopic equations for the $\varepsilon \mathrm{T}_{d}$ phase and the chiral $\varepsilon \mathrm{T}$ phase in the presence of a network. While selected aspects of the hydrodynamics of the $\varepsilon \mathrm{T}_{d}$ phase and the chiral $\varepsilon \mathrm{T}$ phase have been presented in refs. [7,26] and in ref. [8], respectively, a comprehensive presentation has not been given.

In both the $\varepsilon \mathrm{T}_{d}$ and the $\varepsilon \mathrm{T}$ phase we have the same set of macroscopic variables. First, the conserved quantities density $\rho$, energy density $\varepsilon$, density of linear momentum $\boldsymbol{g}$, and the concentration $c$ in mixtures. Secondly, the variables $\delta \Gamma_{i}$ associated with the spontaneously broken continuous rotational symmetries.

These hydrodynamic variables are combined here with the theory of dynamic elasticity that is described by a space and time dependent strain tensor field, $\varepsilon_{i j}$, as macroscopic variable. In case of a transient elasticity, this strain field relaxes on the long time scale. The consequences of the approach of transient elasticity for complex fluids such as polymer solutions and melts have been investigated in the linear $[29,30]$ as well as in the nonlinear regime [31-37] for systems ranging from polymer melts and solutions $[29,31-34,36,37]$ over nematic polymers $[30,35]$ to ferrocholesteric gels and elastomers [38]. Quite recently the applicability of the theory of transient elasticity to a large range of rheologically important small and large amplitude experiments has also been demonstrated $[39,40]$.

In the present paper we analyze for the first time in the field of macroscopic dynamic equations the simultaneous presence of octupolar order and of a transient network as macroscopic variables and we discuss in detail the resulting macroscopic consequences.

\subsection{The macroscopic dynamics of the elastic $\varepsilon T_{d}$ phase}

As the $\mathrm{T}_{d}$ phase, the $\varepsilon \mathrm{T}_{d}$ phase is optically isotropic and has no quadrupolar order. To characterize the 3dimensional rotations associated with spontaneously broken rotational symmetries we use suitable combinations of the changes of the order parameter from its equilibrium value, $\delta T_{i j k}=T_{i j k}-T_{i j k}^{e q}$. Taking into account the symmetry and normalization requirements $\delta T_{i j j}=0$ and $\delta T_{i k l} T_{j k l}+T_{i k l} \delta T_{j k l}=0$, we have for the three normalized hydrodynamic variables [7]

$$
\delta \Gamma_{i} \equiv \frac{1}{4 \alpha} \epsilon_{i p q} T_{p k l} \delta T_{q k l} .
$$

We note that eq. (10) is not integrable.

Since finite 3-dimensional rotations about orthogonal axes in space do not commute, two subsequent operations using $\delta \Gamma_{i}$ lead to $[7]$

$$
\begin{aligned}
\left(\delta_{1} \delta_{2}-\delta_{2} \delta_{1}\right) \Gamma_{i} & =\frac{1}{2 \alpha} \epsilon_{i p q}\left(\delta_{1} T_{p j k}\right)\left(\delta_{2} T_{q j k}\right) \\
& =2 \epsilon_{i p q}\left(\delta_{1} \Gamma_{p}\right)\left(\delta_{2} \Gamma_{q}\right) .
\end{aligned}
$$


This type of relations is well known for the nonlinear hydrodynamics of other condensed matter systems with spontaneously broken rotational symmetries including superfluid ${ }^{3} \mathrm{He}-\mathrm{A}$ (Mermin-Ho relation [41]), biaxial nematics $[42,43]$ and superfluid biaxial nematic models for the core of neutron stars [44].

Finally, there are additional macroscopic variables associated with relative rotations between rotations of the network denoted by $\Omega_{i}$, and the rotations associated with tetrahedral order, $\delta \Gamma_{i}$

$$
J_{i}=\delta \Gamma_{i}-\Omega_{i}
$$

provided the relative orienting energy is small enough. We note that for rigid combined rotations of the two structures $\delta \Gamma_{i}=\Omega_{i}$. The definition of relative rotations just given is suitable for a linearized theory. A generalization to nonlinear relative rotations can be done along the lines described for nematic elastomers [45].

While relative rotations between octupolar order and a (transient or permanent) network have not been considered before, the concept of relative rotations goes back to nematic gels and has been pioneered by de Gennes [46]. Subsequently it has been applied to uniaxial magnetic gels [47] as well as to cholesteric gels and elastomers $[38,48,49]$.

In closing this discussion we emphasize that in a $\varepsilon \mathrm{T}_{d}$ phase we have as invariants, in addition to the Kronecker delta, $\delta_{i j}$, and the totally antisymmetric symbol, $\epsilon_{i j k}$, the tensor $T_{i j k}$ reflecting octupolar order and broken parity symmetry.

\subsubsection{Statics of the $\varepsilon \mathrm{T}_{d}$ phase}

We assume that the first law of thermodynamics is valid locally and thus obtain for the Gibbs relation [50-53] relating the entropy density to changes in all macroscopic variables discussed above

$$
\begin{aligned}
\mathrm{d} \varepsilon= & T \mathrm{~d} \sigma+\mu \mathrm{d} \rho+\mu_{c} \mathrm{~d} c+v_{i} \mathrm{~d} g_{i}+E_{i} \mathrm{~d} D_{i} \\
& +\psi_{i j} \mathrm{~d} \varepsilon_{i j}+L_{i} \mathrm{~d} J_{i}+h_{i}^{\Gamma} \mathrm{d} \Gamma_{i}
\end{aligned}
$$

where $\mathrm{d} \Gamma_{i}=\frac{1}{4 \alpha} \epsilon_{i p q} T_{p k l} \mathrm{~d} T_{q k l}$.

In eq. (13) the thermodynamic forces, temperature $T$, chemical potential $\mu$, relative chemical potential $\mu_{c}$, electric field $E_{i}$, velocity $v_{i}$, the elastic stress $\psi_{i j}$, the "relative molecular field" $L_{i}$ associated with relative rotations and the molecular field $h_{i}^{\Gamma}$ associated with rotations of the octupolar order are defined as variational derivatives of the energy density with respect to the variables while keeping all other variables fixed [53].

Taking into account symmetry arguments including the behavior under time reversal symmetry, parity, rigid rotations and Galilei transformations we obtain for the energy density Legendre transformed with respect to the electric displacement field, $D_{i}$, in harmonic approximation

$$
\begin{aligned}
\varepsilon= & \varepsilon_{0}+\frac{1}{2} c_{i j k l} \varepsilon_{i j} \varepsilon_{k l}+\frac{1}{2} D_{1} J_{i} J_{i} \\
& +\frac{1}{2} K_{i j k l}^{\Gamma}\left(\nabla_{j} \Gamma_{i}\right)\left(\nabla_{l} \Gamma_{k}\right) \\
& +\left(\chi^{\rho} \delta \rho+\chi^{\sigma} \delta \sigma+\chi^{c} \delta c\right) \varepsilon_{i i} \\
& +\tilde{\xi} \epsilon_{i l m} T_{i j k} \varepsilon_{j k} \nabla_{l} \Gamma_{m} \\
& +T_{i j k}\left(\tau^{\rho} \nabla_{k} \rho+\tau^{\sigma} \nabla_{k} \sigma+\tau^{c} \nabla_{k} c\right) \varepsilon_{i j} \\
& +2 \tau^{\Gamma \varepsilon} \epsilon_{i j k}\left(\nabla_{j} \Gamma_{k}\right)\left(\nabla_{l} \varepsilon_{i l}\right) \\
& +\chi E_{i} E_{i}+\zeta T_{i j k} E_{i} \varepsilon_{j k}-\frac{1}{2} \zeta^{E} T_{i j k} E_{i} E_{j} E_{k}
\end{aligned}
$$

with $K_{i j k l}^{\Gamma}=K_{1}^{\Gamma}\left(\delta_{i j} \delta_{k l}+\delta_{i l} \delta_{j k}\right)+K_{2}^{\Gamma} \delta_{i k} \delta_{j l}+K_{3}^{\Gamma} T_{j l p} T_{i k p}$ and where $\varepsilon_{0}$ contains all the terms characteristic of a miscible binary fluid mixture [53] and where $c_{i j k l}$ contains three elastic moduli as is usual for cubic systems [28].

Inspecting eq. (14) several interesting features emerge. While volume strains $\left(\sim \varepsilon_{i i}\right)$ couple directly to variations of density, entropy density and concentrations, other strain components couple to gradients of density, entropy density and concentration. The latter type of coupling is allowed in an octupolar phase while it is forbidden in ordinary isotropic systems due to the absence of broken parity symmetry. The term $\sim \tilde{\xi}$ in eq. (14) is the hydrostatic analogue of the term $\sim \xi$ given in eq. (6). The term $\sim \tau^{\Gamma \varepsilon}$ represents a coupling between gradients of strain and the curl of $\delta \Gamma_{i}$ and is thus already fairly high in gradients. It is also worth noting that the contribution associated with relative rotations couples statically to no other macroscopic variable, a feature that can be traced back to the isotropy of the system studied here. As we will show in the next subsection on dynamics, dissipative cross-coupling terms to relative rotations can emerge even for an optically isotropic system.

The thermodynamic forces are obtained by taking the appropriate variational derivatives of eq. (14) with respect to one variable, where the ellipses indicate that all other variables are fixed [53]. We obtain in harmonic approximation

$$
\begin{gathered}
v_{i}=\left.\frac{\partial \varepsilon}{\partial g_{i}}\right|_{\ldots}=\frac{1}{\rho} g_{i}, \\
\delta \mu=\left.\frac{\delta \varepsilon}{\delta(\delta \rho)}\right|_{\ldots}=\chi^{\rho} \varepsilon_{i i}-\nabla_{k}\left(T_{i j k} \tau^{\rho} \varepsilon_{i j}\right), \\
\delta T=\left.\frac{\delta \varepsilon}{\delta(\delta \sigma)}\right|_{\ldots}=\chi^{\sigma} \varepsilon_{i i}-\nabla_{k}\left(T_{i j k} \tau^{\sigma} \varepsilon_{i j}\right), \\
\delta \mu_{c}=\left.\frac{\delta \varepsilon}{\delta(\delta c)}\right|_{\ldots}=\chi^{c} \varepsilon_{i i}-\nabla_{k}\left(T_{i j k} \tau^{c} \varepsilon_{i j}\right), \\
L_{i}=\left.\frac{\partial \epsilon}{\partial J_{i}}\right|_{\ldots}=D_{1} J_{i}, \\
P_{i}=\left.\frac{\partial \epsilon}{\partial E_{i}}\right|_{\ldots}=\chi E_{i}-\frac{2}{3} T_{i j k} E_{j} E_{k},
\end{gathered}
$$




$$
\begin{aligned}
\psi_{i j}= & \left.\frac{\delta \epsilon}{\delta \varepsilon_{i j}}\right|_{\ldots}=c_{i j k l} \varepsilon_{k l}+\delta_{i j}\left(\chi^{\rho} \delta \rho+\chi^{\sigma} \delta \sigma+\chi^{c} \delta c\right) \\
& +T_{i j k}\left(\tau^{\rho} \nabla_{k} \rho+\tau^{\sigma} \nabla_{k} \sigma+\tau^{c} \nabla_{k} c\right) \\
& +\tilde{\xi} \epsilon_{k l m} T_{k i j} \nabla_{l} \Gamma_{m} \\
& -\tau^{\Gamma \varepsilon}\left(\epsilon_{i k l} \nabla_{j}+\epsilon_{j k l} \nabla_{i}\right) \nabla_{k} \Gamma_{l} \\
h_{i}^{\Gamma}= & \left.\frac{\delta \epsilon}{\delta \Gamma_{i}}\right|_{\ldots}=-\nabla_{j}\left[K_{i j k l}^{\Gamma}\left(\nabla_{l} \Gamma_{k}\right)\right]-\tilde{\xi} \epsilon_{m l i} \nabla_{l}\left(T_{m j k} \varepsilon_{j k}\right) \\
& -2 \tau^{\Gamma \varepsilon_{k j i}} \epsilon_{j} \nabla_{l} \varepsilon_{k l} .
\end{aligned}
$$

We note that in the nonlinear domain $h_{i}^{\Gamma}$ picks up a contribution from the noncommutativity relation, eq. (11) compare ref. [9].

\subsubsection{Dynamics of the $\varepsilon \mathrm{T}_{d}$ phase}

The macroscopic equations for conserved quantities, variables associated with spontaneously broken continuous symmetries and macroscopic variables take, in the $\varepsilon \mathrm{T}_{d}$ phase, the form

$$
\begin{aligned}
& \frac{\partial}{\partial t} \rho+\operatorname{div} \rho \boldsymbol{v}=0, \\
& \left(\frac{\partial}{\partial t}+v_{i} \nabla_{i}\right) \rho^{e l}+\operatorname{div} \boldsymbol{j}^{e l}=0, \\
& \frac{\partial}{\partial t} \sigma+\operatorname{div} \sigma \boldsymbol{v}+\operatorname{div} \boldsymbol{j}^{\sigma}=\frac{2 R}{T}, \\
& \frac{\partial}{\partial t} g_{i}+\nabla_{j}\left(v_{j} g_{i}+\delta_{i j} \Pi-\psi_{i j}+\sigma_{i j}^{t h}+\sigma_{i j}\right)=0, \\
& \rho\left(\frac{\partial}{\partial t}+v_{j} \nabla_{j}\right) c+\operatorname{div} \boldsymbol{j}^{c}=0, \\
& \left(\frac{\partial}{\partial t}+v_{j} \nabla_{j}\right) J_{i}+Y_{i}^{\Omega}=0, \\
& \left(\frac{\partial}{\partial t}+v_{k} \nabla_{k}\right) \varepsilon_{i j}-A_{i j} \\
& \quad+\varepsilon_{k j} \nabla_{i} v_{k}+\varepsilon_{k i} \nabla_{j} v_{k}+X_{i j}^{\varepsilon}=0, \\
& \left(\frac{\partial}{\partial t}+v_{j} \nabla_{j}\right) \Gamma_{i}+Y_{i}=0
\end{aligned}
$$

with the momentum density $g_{i}=\rho v_{i}$, the electric charge density $\rho^{e l}=(1 / 4 \pi) \nabla_{i} D_{i}$, and $[32,54]$ the thermodynamic part of the stress tensor

$$
\begin{aligned}
\sigma_{i j}^{t h}= & -E_{j} D_{i}+2 \psi_{j k} \varepsilon_{k i}-\frac{1}{2}\left(J_{j} L_{i}-J_{i} L_{j}\right) \\
& -\frac{1}{2}\left(\delta \Gamma_{j} h_{i}^{\Gamma}-\delta \Gamma_{i} h_{j}^{\Gamma}\right),
\end{aligned}
$$

where $A_{i j}=\frac{1}{2}\left(\nabla_{i} v_{j}+\nabla_{j} v_{i}\right)$ is the deformational flow and $\omega_{i}=\frac{1}{2} \epsilon_{i j k} \nabla_{j} v_{k}$ the vorticity. The thermodynamic pressure $\Pi$ is given by

$$
\Pi=-\epsilon+T \sigma+\mu \rho+\mu_{c} c+\boldsymbol{g} \cdot \boldsymbol{v}+\boldsymbol{E} \cdot \boldsymbol{D} .
$$

The parts of the currents shown explicitly in (23)-(31) are not material dependent, but are given by general symmetry and thermodynamic principles [53], like the transformation behavior under translations (transport terms) or rotations (convective terms) and by the requirement of zero entropy production $(R=0)$ of all those terms together with the isotropic pressure term in eq. (26).

Using the requirement [53]

$$
\omega_{i j}\left(-P_{i} E_{j}+L_{i} J_{j}+h_{i}^{\Gamma} \delta \Gamma_{j}+2 \psi_{k i} \varepsilon_{k j}\right)=0
$$

for any constant antisymmetric matrix $\omega_{i j}=-\omega_{j i}$, which ensures the rotational invariance of the Gibbs relation. With eq. (33) the nonsymmetric part of the stress tensor, eq. (31), can be removed and

$$
2 \sigma_{i j}^{t h}=-\left(E_{j} D_{i}+E_{i} D_{j}\right)+2\left(\psi_{j k} \varepsilon_{k i}+\psi_{i k} \varepsilon_{k j}\right)
$$

is manifestly symmetric. When the energy depends on gradients of the strain, there is an additional contribution on the right-hand side of eq. (34), $\psi_{l j k} \nabla_{l} \varepsilon_{i k}+\psi_{l i k} \nabla_{l} \varepsilon_{j k}$ with $\psi_{i j k} \equiv(\partial \varepsilon) /\left(\partial \nabla_{k} \varepsilon_{i j}\right)$, which is usually neglected for transient networks. The origin of such a stress contribution is similar to that of the Ericksen stress in nematic liquid crystals [53].

The source term $2 R / T$ in (25) is the entropy production, which has to be zero for reversible, and positive for irreversible processes. The phenomenological parts of the entropy current $j_{i}^{\sigma}$, the stress tensor $\sigma_{i j}$, the concentration current $j_{i}^{c}$ and the quasi-currents $Y_{i}^{\Omega}, Y_{i}$, and $X_{i j}^{\varepsilon}$, associated with the temporal changes of relative rotations, the tetrahedral rotations, and the strain tensor, respectively, are given below. These phenomenological currents and quasi-currents can be split into reversible (superscript $R$ ) and dissipative parts (superscript $D$ ), where the former have the same time reversal behavior as the time derivative of the appropriate variable and must give $R=0$, while the latter have the opposite behavior and give $R>0$. The phenomenological currents and quasi-currents are given within "linear irreversible thermodynamics" (guaranteeing general Onsager relations), i.e. as linear relations between currents and thermodynamic forces. The resulting expressions are nevertheless nonlinear, since all material parameters can be functions of the state variables $(e . g ., \rho$, $T)$. The phenomenological part of the stress tensor $\sigma_{i j}$ has to be symmetric guaranteeing angular momentum conservation. The dynamic equation for the energy density follows from eqs. (23)-(29) via the Gibbs relation eq. (13), and is not shown here.

\subsubsection{Reversible currents in the $\varepsilon \mathrm{T}_{d}$ phase}

Making use of symmetry arguments (including behavior under time reversal, parity, rigid rotations, rigid translations and covariance under Galilei transformations) and of Onsager's relations we obtain the following expressions for the reversible currents up to linear order in the thermodynamic forces:

$$
\begin{aligned}
g_{i} & =\rho v_{i}, \\
j_{i}^{e l R} & =\Gamma_{1} T_{i j k} A_{j k}, \\
j_{i}^{\sigma R} & =\Gamma_{2} T_{i j k} A_{j k},
\end{aligned}
$$




$$
\begin{aligned}
j_{i}^{c R}= & \Gamma_{3} T_{i j k} A_{j k}, \\
X_{i j}^{\varepsilon R}= & \frac{1}{2} \Gamma_{4}\left(T_{i k l} \nabla_{j}+T_{j k l} \nabla_{i}\right) A_{k l}, \\
\sigma_{i j}^{R}= & -\Gamma_{1} T_{k j i} E_{k}-\Gamma_{2} T_{k j i} \nabla_{k} T-\Gamma_{3} T_{k j i} \nabla_{k} \mu_{c} \\
& +\Gamma_{4} T_{k j i} \nabla_{l} \psi_{k l}, \\
Y_{i}^{R}= & -\frac{1}{2} \omega_{i}+\frac{1}{2 \alpha} \epsilon_{i p q} \epsilon_{m j l} T_{p j k} T_{q l k} \omega_{m}, \\
Y_{i}^{\Omega R}= & 0 .
\end{aligned}
$$

We have kept only one new term $\left(\sim \Gamma_{4}\right)$ related to a network, neglecting two other ones of the form $T_{k j l} \nabla_{i} \psi_{k l}$ and $T_{k l j} \nabla_{l} \psi_{k i}$. All involve only gradients of the elastic stresses, rather than the elastic stresses themselves. Secondly, there is also no dynamic reversible cross-coupling term between relative rotations and velocity gradients for parity reasons. We note that the coupling terms between gradients of the entropy density and of concentration as well as of electric fields, on the one hand, and symmetrized velocity gradients, on the other, are isomorphic to those given in ref. [26]. The structure of eq. (41) has been presented in ref. [7] for the $\mathrm{T}_{d}$ phase in the absence of a network.

\subsubsection{Irreversible currents and entropy production in the $\varepsilon \mathrm{T}_{d}$ phase}

For the derivation of the dissipative parts of the phenomenological currents one usually expands the dissipation function $R$ to second order in the thermodynamic forces and then obtains the dissipative currents by taking the variational derivatives with respect to the forces. Note that for a permanent network gradients of the elastic stress, $\nabla_{k} \psi_{i j}$, are the thermodynamic forces, while for a transient network also the stresses, $\psi_{i j}$, are thermodynamic forces. We find for the dissipation function

$$
\begin{aligned}
R= & \frac{1}{2} \kappa\left(\nabla_{i} T\right)\left(\nabla_{i} T\right)+\frac{1}{2} \nu_{i j k l} A_{i j} A_{k l}+\left(\frac{1}{2 \tau}\right)_{i j k l} \psi_{i j} \psi_{k l} \\
& +\frac{1}{2} \gamma\left(\nabla_{k} \psi_{i k}\right)\left(\nabla_{l} \psi_{i l}\right)+\frac{1}{2} D\left(\nabla_{i} \mu_{c}\right)\left(\nabla_{i} \mu_{c}\right)+\frac{1}{2} \sigma E_{i} E_{i} \\
& +D^{T}\left(\nabla_{i} T\right)\left(\nabla_{i} \mu_{c}\right)+D^{E} E_{i} \nabla_{i} \mu_{c}+\kappa^{E} E_{i} \nabla_{i} T \\
& +\zeta^{E} E_{i} \nabla_{k} \psi_{i k}+\left(\nabla_{j} \psi_{i j}\right)\left(\zeta^{T} \nabla_{i} T+\zeta^{c} \nabla_{i} \mu_{c}+\zeta^{E} E_{i}\right) \\
& +\frac{1}{2 \gamma_{1}} h_{i}^{\Gamma} h_{i}^{\Gamma}+\frac{1}{2} \zeta L_{i} L_{i}+\zeta_{12} L_{i} h_{i}^{\Gamma} \\
& +T_{i j k} \psi_{j k}\left(\xi^{T} \nabla_{i} T+\xi^{c} \nabla_{i} \mu_{c}+\xi^{E} E_{i}\right) .
\end{aligned}
$$

Here the two fourth rank tensors $\nu_{i j k l}$ and $(1 / \tau)_{i j k l}$ are of the form characteristic for cubic systems with the same symmetry as that for the elastic moduli [28] containing 3 independent coefficients, each. The coefficients $\kappa, D, D^{T}$, $\sigma$ and $\kappa^{E}$ describe (isotropic) heat conduction, mass diffusion, thermodiffusion, electric conductivity and the Peltier effect, respectively. The strain diffusion $(\sim \gamma)$ has been simplified to a scalar coupling, while in the general case a rank-6 material tensor with 9 coefficients is involved. $\gamma_{1}$ is associated with the diffusion of the tetrahedral vector, $\zeta$ characterizes the relaxation of relative rotations and the contribution $\sim \zeta_{12}$ represents the coupling between relative rotations and diffusion of the tetrahedral orientations. The three contributions are in structure similar to the case of nematic elastomers [54]. We note, however, that in the present tetrahedral case $h_{i}^{\Gamma}$ and $L_{i}$ have three components each.

Inspecting carefully eq. (43) one notes that in lowest order in the wave vector relative rotations and tetrahedral angles show no dissipative cross-coupling terms to other variables, while they are coupling to each other.

The last line contains direct dissipative cross-coupling terms of elastic stresses to gradients of temperature and relative chemical potential as well as to electric fields. These terms are absent for other optically isotropic systems for reasons of parity.

The range of possible values of the coefficients in eq. (43) is restricted by the positivity of the entropy production.

To obtain the dissipative parts of the currents and quasi-currents we take the variational derivatives of $R$ with respect to the appropriate thermodynamic force

$$
\begin{aligned}
j_{i}^{\sigma D}= & -\kappa \nabla_{i} T-D^{T} \nabla_{i} \mu_{c}-\kappa^{E} E_{i} \\
& -\zeta^{T} \nabla_{k} \psi_{i k}-\xi^{T} T_{i j k} \psi_{j k} \\
j_{i}^{c D}= & -D \nabla_{i} \mu_{c}-D^{T} \nabla_{i} T-D^{E} E_{i} \\
& -\zeta^{c} \nabla_{k} \psi_{i k}-\xi^{c} T_{i j k} \psi_{j k} \\
j_{i}^{e l D}= & \sigma E_{i}+D^{E} \nabla_{i} \mu_{c}+\kappa^{E} \nabla_{i} T \\
& +\zeta^{E} \nabla_{k} \psi_{i k}+\xi^{E} T_{i j k} \psi_{j k} \\
\sigma_{i j}^{D}= & -\nu_{i j k l}^{D} A_{k l}, \\
Y_{i}^{D}= & \frac{1}{\gamma_{1}} h_{i}^{\Gamma}+\zeta_{12} L_{i} \\
Y_{i}^{\Omega D}= & \zeta L_{i}^{\perp}+\zeta_{12} h_{i}^{\Gamma} \\
X_{i j}^{\varepsilon D}= & \left(\frac{1}{\tau}\right)_{i j k l} \psi_{k l}-\frac{\gamma}{2} \nabla_{k}\left(\nabla_{j} \psi_{i k}+\nabla_{i} \psi_{j k}\right) \\
& +T_{k i j}\left(\xi^{T} \nabla_{k} T+\xi^{c} \nabla_{k} \mu_{c}+\xi^{E} E_{k}\right) \\
& -\zeta^{T} \nabla_{i} \nabla_{j} T-\zeta^{c} \nabla_{i} \nabla_{j} \mu_{c}-\frac{\zeta^{E}}{2}\left(\nabla_{i} E_{j}+\nabla_{j} E_{i}\right)
\end{aligned}
$$

\subsection{The macroscopic dynamics of the elastic chiral $\varepsilon T$ phase}

In this section we discuss chiral contributions, which arise when, in addition to the tetrahedral/octupolar order, $T_{i j k}$, a pseudoscalar quantity, $q_{0}$, is present. Several aspects of the $\mathrm{T}$ phase, both microscopic and macroscopic, have been discussed in the appendix of ref. [8]. Here we focus on the macroscopic aspects and, in particular, on the coupling terms to a network and to relative rotations in the chiral $\varepsilon \mathrm{T}$ phase.

Clearly, the macroscopic variables in the $\varepsilon \mathrm{T}_{d}$ and in the $\varepsilon \mathrm{T}$ phase are the same. For the additional static contributions in the $\varepsilon \mathrm{T}$ phase due to the existence of a 
pseudoscalar quantity we have

$$
\begin{aligned}
\varepsilon_{T}= & \varepsilon_{T_{d}}+q_{0} K_{1}^{l i n} \nabla_{i} \Gamma_{i} \\
& +q_{0}\left(\xi^{\rho} \delta \rho+\xi^{\sigma} \delta \sigma+\xi^{c} \delta c\right) \nabla_{i} \Gamma_{i} \\
& +q_{0} \xi_{i j k l}\left(\nabla_{i} \Gamma_{j}\right) \varepsilon_{k l} \\
& +D_{2} q_{0} T_{i j k} J_{i} \varepsilon_{j k}+\zeta^{R} q_{0} J_{i} E_{i}
\end{aligned}
$$

where $\xi_{i j k l}=\xi_{1} \delta_{i j} \delta_{k l}+\xi_{2}\left(\delta_{i k} \delta_{j l}+\delta_{i l} \delta_{j k}\right)$ and with one linear chiral term $\sim q_{0} K_{1}^{\text {lin }}$ whose consequences have been already sketched out in ref. [8]. The optimum helical pitch, different from the chiral pseudoscalar of the phase, $q_{0}$, since there is no a priori reason that $K_{1}^{l i n}$ is related to $K_{1,2}^{T}[8]$.

An analogous statement holds for ordinary cholesterics [55]. Helical rotations about the 2-fold axes do not lower the free energy, since the linear gradient term is zero in that case and the quadratic term, $\sim K_{3}$, increases the free energy. The second line of eq. (51) contains terms [8] similar to the static Lehmann effect of ordinary cholesterics [56-60]. The contribution in the third line represents a coupling between strains and spatial variations of the octupolar order. We note that it has a different structure from the coupling $\sim \tilde{\xi}$ in eq. (14). The are two static crosscoupling terms to relative rotations while there was none in the nonchiral case. The coupling $\sim D_{2}$ between strains and relative rotations has no analogue for any other known optically isotropic system. The contribution $\sim \zeta^{R}$ can be viewed as the analogue of rotato-electricity as studied before for cholesteric elastomers and gels $[48,49,60]$. What is worth emphasizing about the effect given here is the fact that it gives rise to an effect linear in an external electric field in an optically isotropic system (before the field is applied).

Next we give the additional chiral contributions to the thermodynamic conjugate quantities that arise from the chiral energy by taking the variational derivative with respect to the appropriate variables

$$
\begin{aligned}
\delta T= & -q_{0} \xi_{\sigma}\left(\nabla_{i} \Gamma_{i}\right), \\
\delta \mu= & -q_{0} \xi_{\rho}\left(\nabla_{i} \Gamma_{i}\right), \\
\delta \mu_{c}= & -q_{0} \xi_{c}\left(\nabla_{i} \Gamma_{i}\right), \\
\psi_{i j}= & q_{0} \xi_{k l i j}\left(\nabla_{k} \Gamma_{l}\right)+D_{2} q_{0} T_{k i j} J_{k}, \\
L_{i}= & +q_{0} D_{2} T_{i j k} \varepsilon_{j k}+\zeta^{R} q_{0} E_{i}, \\
D_{i}= & q_{0} \zeta^{R} J_{i}, \\
h_{i}^{\Gamma}= & -q_{0} \nabla_{i}\left(\xi_{c} c+\xi_{\sigma} \sigma+\xi_{\rho} \rho\right) \\
& -q_{0} \nabla_{j}\left(\xi_{j i k l} \varepsilon_{k l}\right) .
\end{aligned}
$$

Now we turn to a discussion of the dynamic chiral contributions. Using the condition $R=0$ and the general symmetry arguments outlined above, we obtain the following expressions for the chiral parts of the reversible currents up to linear order in the thermodynamic forces:

$$
\begin{aligned}
j_{i}^{\sigma R} & =0, \\
j_{i}^{c R} & =0, \\
j_{i}^{e l R} & =0, \\
X_{i j}^{\varepsilon R} & =0, \\
\sigma_{i j}^{R} & =q_{0} T_{i j k}\left(\lambda h_{k}^{\Gamma}+\lambda^{J} L_{k}\right), \\
Y_{i}^{R} & =q_{0} \lambda T_{i j k} A_{j k}, \\
Y_{i}^{\Omega R} & =q_{0} \lambda^{J} T_{i j k} A_{j k},
\end{aligned}
$$

where we have disregarded gradients of the thermodynamic forces.

As has been shown in refs. [8,9] the chiral contribution in the quasi-current for rotations of the tetrahedra combines with the behavior under rigid rotations of the tetrahedra (compare eq. (41) from the section on the $\varepsilon \mathrm{T}_{d}$ phase) to allow for a stationary alignment of $T_{i j k}$ in simple shear independent of the flow rate and only dependent on the product $q_{0} \lambda$. This scenario arises if one of the 3fold tetrahedral axes is in the vortex direction $[8,9]$, while there is no flow alignment of a 2-fold axis under shear.

We emphasize that the flow alignment just discussed is not possible for relative rotations since they do not have a coupling to rotational flow (compare eq. (42)).

The dissipative part of the dynamics for the $\varepsilon \mathrm{T}$ phase with transient elasticity associated with the presence of the pseudoscalar $q_{0}$, can be discussed most succinctly in terms of the dissipation function. For the dissipation function of the $\varepsilon \mathrm{T}$ we get in addition to eq. (43)

$$
\begin{aligned}
R_{T}= & q_{0} h_{k}^{\Gamma}\left(\psi^{c} \nabla_{k} \mu_{c}+\psi^{\sigma} \nabla_{k} T+\psi^{e} E_{k}\right) \\
& +q_{0} L_{k}\left(\psi^{c J} \nabla_{k} \mu_{c}+\psi^{\sigma J} \nabla_{k} T+\psi^{e J} E_{k}\right) \\
& +q_{0} \psi_{i j} T_{i j k}\left(\chi^{\Gamma \psi} h_{k}^{\Gamma}+\chi^{J \psi} L_{k}\right) .
\end{aligned}
$$

It contains all the dissipative Lehmann-type effects relating gradients of temperature and concentration, and electric fields to rotations of tetrahedral order (first line) and relative rotations (second line). In contrast to the static case, where there is no coupling to an electric field [56], such a contribution arises naturally in the dissipation function. On the other hand, there is no such dissipative coupling to density gradients, since there is no dissipative current associated with density (due to mass conservation). The third line describes appropriate dissipative couplings of tetrahedral rotations and relative rotations to elastic deformations not considered before for systems with tetrahedral order. Some of their consequences will be elucidated in the next section.

We stress that these dissipative contributions are specific for the $\varepsilon \mathrm{T}$ phase with a transient network. They do not arise for the $\varepsilon \mathrm{T}_{d}$ phase and also not for permanently cross-linked systems without transient elasticity, since in that case only gradients of the elastic stresses are allowed to enter the dissipation function. 
The chiral parts of the dissipative currents then read

$$
\begin{aligned}
j_{i}^{\sigma D}= & -q_{0}\left(\psi^{T} h_{k}^{\Gamma}+\psi^{T J} L_{k}\right), \\
j_{i}^{c D}= & -q_{0}\left(\psi^{c} h_{k}^{\Gamma}+\psi^{c J} L_{k}\right), \\
j_{i}^{e l D}= & -q_{0}\left(\psi^{e} h_{j}^{\Gamma}+\psi^{e J} L_{k}\right), \\
\sigma_{i j}^{D}= & 0, \\
Y_{i}^{D}= & q_{0}\left(\psi_{c} \nabla_{k} \mu_{c}+\psi_{\sigma} \nabla_{k} T+\psi_{e} E_{k}\right) \\
& +q_{0} T_{i j k} \chi^{\Gamma \psi} \psi_{j k}, \\
Y_{i}^{\Omega D}= & q_{0}\left(\psi^{c J} \nabla_{k} \mu_{c}+\psi^{\sigma J} \nabla_{k} T+\psi^{e J} E_{k}\right) \\
& +q_{0} T_{i j k} \chi^{J \psi} \psi_{j k}, \\
X_{i j}^{\epsilon D}= & q_{0} T_{i j k}\left(\chi^{J \psi} h_{k}^{\Gamma}+\chi^{\Gamma \psi} L_{k}\right),
\end{aligned}
$$

totaling 8 purely chiral dissipative transport parameters.

As in the reversible case we have refrained from discussing effects involving gradients of the thermodynamic forces.

\section{Suggestions for experiments}

\subsection{Considerations for the $\varepsilon T_{d}$ phase}

First, we discuss briefly the question of reversible crosscoupling terms. Analyzing all reversible cross-coupling arising in the $\varepsilon \mathrm{T}_{d}$ that are listed in sect. 3.1.3 most of them have been presented before $[9,26]$ for the $\mathrm{T}_{d}$ phase without a network. Only the terms $\sim \Gamma_{4}$ in eqs. (39) and (40) are related to the network and describe stresses induced by gradients of elastic strains as well as temporal changes of strains due to inhomogeneous flow. However these terms are of higher order in gradients compared to the ordinary elastic couplings and are therefore hardly suitable for measurements.

Inspecting the various static cross-coupling terms in eq. (14), we find that there is one class of terms which is characteristic for a $\varepsilon \mathrm{T}_{d}$ phase with a network. To lowest order in the gradients, there are coupling terms between off-diagonal components of the strain and gradients of density, concentrations and entropy density. Specializing eqs. (16)-(18) to this case we find in linear order

$$
\begin{aligned}
\delta \mu & =\ldots-\tau^{\rho} T_{i j k} \nabla_{k} \varepsilon_{i j}, \\
\delta T & =\ldots-\tau^{\sigma} T_{i j k} \nabla_{k} \varepsilon_{i j}, \\
\delta \mu_{c} & =\ldots-\tau^{c} T_{i j k} \nabla_{k} \varepsilon_{i j} .
\end{aligned}
$$

These coupling terms lead to static changes of temperature, chemical potential and osmotic chemical potential induced by strain gradients, irrespective of whether the strains are transient or permanent, provided the material has octupolar order.

We note that the piezoelectric term listed in eq. (20) has been discussed already in the section on GinzburgLandau consideration for the $\varepsilon \mathrm{T}_{d}$ phase (sect. 2.1).

Inspecting eq. (43) for the dissipation function of the $\varepsilon \mathrm{T}_{d}$ phase with a network we see that there are direct dissipative coupling terms between transient stresses $\psi_{i j}$ on the one hand and temperature gradients, gradients of the relative chemical potential and electric fields on the other. Specializing eqs. (44)-(46) to these cross-coupling terms we obtain

$$
\begin{aligned}
j_{i}^{\sigma D} & =\ldots-\xi^{T} T_{i j k} \psi_{j k} \\
j_{i}^{c D} & =\ldots-\xi^{c} T_{i j k} \psi_{j k}, \\
j_{i}^{e l D} & =\ldots+\xi^{E} T_{i j k} \psi_{j k}
\end{aligned}
$$

Thus elastic stresses of a transient network produce dissipative electric currents, heat currents and concentration currents. Conversely, temperature gradients, concentration gradients and electric fields lead to temporal variations of the transient network

$$
X_{i j}^{\varepsilon D}=\ldots+T_{k i j}\left(\xi^{T} \nabla_{k} T+\xi^{c} \nabla_{k} \mu_{c}+\xi^{E} E_{k}\right) .
$$

In addition, there are dissipative crosscouplings involving gradients of elastic stresses that lead to heat, concentration and electric currents. Vice versa, second-order gradients of temperature or osmotic chemical potential and electric field gradients generate temporal changes of the network. These couplings are operative for permanent networks as well (cf. appendix).

\subsection{Considerations for the chiral $\varepsilon \mathrm{T}$ phase}

In the chiral $\varepsilon \mathrm{T}$ phase the pseudoscalar $q_{0}$ allows for additional cross-couplings when compared to the $\varepsilon \mathrm{T}_{d}$ phase.

In refs. $[8,9]$ it has already been pointed out for the $\mathrm{T}$ phase without a network that reversible flow alignment of the tetrahedral orientations is possible - in contrast to the case of the $\mathrm{T}_{d}$ phase. In the presence of a network there is an additional remarkable reversible cross-coupling term between relative rotations and shear stresses. Specializing eqs. (63) and (65) we have

$$
\begin{aligned}
\sigma_{i j}^{R} & =\ldots+q_{0} T_{i j k} \lambda^{J} L_{k}, \\
Y_{i}^{\Omega R} & =\ldots+q_{0} T_{i j k} \lambda^{J} A_{j k} .
\end{aligned}
$$

Equations (81) and (82) show that relative rotations generate shear stress and that extensional flow produces temporal changes of the relative rotations. For the $\varepsilon \mathrm{T}_{d}$ phase these effects are not possible for parity reasons.

The most interesting static coupling terms in the $\varepsilon \mathrm{T}$ phase induced by the existence of $q_{0}$ are the two coupling terms between relative rotations and the network or an electric field, respectively, as given in the last line of eq. (51).

Specifically, we find for the associated thermodynamic forces of interest, namely the elastic stress, the thermodynamic force connected to relative rotations, and the electric field, respectively

$$
\begin{aligned}
\psi_{i j} & =\ldots+q_{0} D_{2} T_{k i j} J_{k}, \\
L_{i} & =\ldots+q_{0} D_{2} T_{i j k} \varepsilon_{j k}+q_{0} \zeta^{R} E_{i}, \\
D_{i} & =\ldots+q_{0} \zeta^{R} J_{i} .
\end{aligned}
$$


From eqs. (84) and (85) we see that the $\varepsilon \mathrm{T}$ phase features the analogue of rotatoelectricity, well established for cholesteric and chiral smectic elastomers [48, 49, 60]. Applied electric fields lead to relative rotations and, vice versa, relative rotations lead to the occurrence of an electric field. From eqs. (83) and (84) we conclude that in the $\varepsilon \mathrm{T}$ phase relative rotations generate elastic stresses and strains produce relative rotations. This coupling, $\sim D_{2}$, is absent for the $\varepsilon \mathrm{T}_{d}$ phase and thus presents one option to distinguish $\varepsilon \mathrm{T}$ and $\varepsilon \mathrm{T}_{d}$ phases although both are optically isotropic.

For the dissipative coupling terms between a transient network and relative rotations in the $\varepsilon \mathrm{T}$ phase we find — specializing eqs. (71)-(73)

$$
\begin{aligned}
Y_{i}^{D} & =\ldots+q_{0} T_{i j k} \chi^{\Gamma \psi} \psi_{j k}, \\
Y_{i}^{\Omega D} & =\ldots+q_{0} T_{i j k} \chi^{J \psi} \psi_{j k}, \\
X_{i j}^{\epsilon D} & =\ldots+q_{0} T_{i j k}\left(\chi^{J \psi} h_{k}^{\Gamma}+\chi^{\Gamma \psi} L_{k}\right) .
\end{aligned}
$$

Thus, elastic stresses lead to rotations of the octupolar/tetrahedral structure and temporal changes of relative rotations, eq. (86) and (87), respectively. Conversely, eq.(88) describes how relative rotations and, e.g., gradients in the orientation of the octupolar structure give rise to dissipative temporal changes of the network. These effects only occur in transient networks.

We close this section by emphasizing that all these effects can only arise for optically isotropic phases with octupolar order, but not for completely isotropic phases.

\section{Summary and conclusions}

Using a Ginzburg-Landau analysis we have shown for the first time that it is possible to obtain chiral domains of opposite hand in an optically isotropic phase with transient elasticity composed of achiral molecules provided the phase shows octupolar order. Thus we have come up with a novel mechanism for chiral symmetry breaking on mesoscopic and macroscopic scales for optically isotropic phases. Therefore the model presented gives a possible explanation for recent experimental results.

In the bulk part of this paper we have presented the macroscopic dynamic equations for optically isotropic tetrahedral phases in the presence of a network. Such a macroscopic dynamic description for the simultaneous presence of octupolar order and a transient network has not been given before. We find that for the nonchiral $\varepsilon \mathrm{T}_{d}$ phase and the chiral $\varepsilon \mathrm{T}$ phase elasticity leads to a number of new cross-coupling terms. These are due, in particular, to static and dissipative dynamic coupling effects.

In the nonchiral $\varepsilon \mathrm{T}_{d}$ phase, strain fields couple statically to gradients of density, entropy density and concentration while dissipatively heat currents, concentration currents and electric currents couple to stresses associated with elasticity.

In the chiral $\varepsilon \mathrm{T}$ phase we find - in addition to the cross-coupling terms already sketched out for the $\varepsilon \mathrm{T}_{d}$ phase - that statically relative rotations couple to the strain field and to electric fields, while dissipatively stresses associated with transient elasticity couple to relative rotations as well as to the molecular field associated with rotations of the octupolar orientations.

Partial support of this work through the Schwerpunktprogramm SPP 1681 "Feldgesteuerte Partikel-MatrixWechselwirkungen: Erzeugung, skalenübergreifende Modellierung und Anwendung magnetischer Hybridmaterialien" of the Deutsche Forschungsgemeinschaft is gratefully acknowledged.

\section{Author contribution statement}

The authors contributed equally to the paper.

\section{Appendix A. Simplifications in the case of a permanent network}

In the previous sections we have outlined the macroscopic behavior of phases with octupolar order for the case that a network is present. The network could have been permanent or transient. The elastic energy, and thus the statics, is the same for both cases. The difference lies in the dynamics, in particular in the dynamics of the strain field, since in the viscoelastic case the strain tensor relaxes, while in the permanent case, the strain can only diffuse. Thus, in the latter case, the current in eq. (29) that allows for relaxation, $X_{i j}^{\varepsilon}$, has to be of the diffusional form $\nabla_{k} X_{i j k}^{\varepsilon}$. If the thermodynamic force that enters the currents is a divergence, $\nabla_{k} \psi_{i k}$, the dynamic equation has the simpler form

$$
\begin{aligned}
& \left(\frac{\partial}{\partial t}+v_{k} \nabla_{k}\right) \varepsilon_{i j}-A_{i j}+\varepsilon_{k j} \nabla_{i} v_{k}+\varepsilon_{k i} \nabla_{j} v_{k} \\
& +\frac{1}{2}\left(\nabla_{j} X_{i}^{\varepsilon}+\nabla_{i} X_{j}^{\varepsilon}\right)=0
\end{aligned}
$$

with an elasticity current $X_{i}^{\varepsilon}=X_{i}^{\varepsilon R}+X_{i}^{\varepsilon D}$.

Due to the importance of the permanent elastic case for solids and crystals as well as to gels and elastomers, we will present the appropriate equations explicitly.

\section{Appendix A.1. The $\varepsilon T_{d}$ phase in the presence of a permanent network}

If only a permanent network is present, this simplifies the dissipation function, eq. (43), since all contributions $\sim \psi_{i j}$ are absent:

$$
\begin{aligned}
R= & \frac{1}{2} \kappa\left(\nabla_{i} T\right)\left(\nabla_{i} T\right)+\frac{1}{2} \nu_{i j k l} A_{i j} A_{k l} \\
& +\frac{1}{2} \gamma\left(\nabla_{k} \psi_{i k}\right)\left(\nabla_{l} \psi_{i l}\right)+\frac{1}{2} D\left(\nabla_{i} \mu_{c}\right)\left(\nabla_{i} \mu_{c}\right)+\frac{1}{2} \sigma E_{i} E_{i} \\
& +D^{T}\left(\nabla_{i} T\right)\left(\nabla_{i} \mu_{c}\right)+D^{E} E_{i} \nabla_{i} \mu_{c}+\kappa^{E} E_{i} \nabla_{i} T \\
& +\zeta^{E} E_{i} \nabla_{k} \psi_{i k}+\left(\nabla_{j} \psi_{i j}\right)\left(\zeta^{T} \nabla_{i} T+\zeta^{c} \nabla_{i} \mu_{c}+\zeta^{E} E_{i}\right) \\
& +\frac{1}{2 \gamma_{1}} h_{i}^{\Gamma} h_{i}^{\Gamma}+\frac{1}{2} \zeta L_{i} L_{i}+\zeta_{12} L_{i} h_{i}^{\Gamma} .
\end{aligned}
$$


This means that the terms $\sim \xi^{T}, \xi^{c}$, and $\xi^{E}$ in the heat, concentration and electric currents, respectively, eqs. (44)-(46), are absent and the dissipative part of the elastic current reads

$$
X_{i}^{\varepsilon D}=-\gamma \nabla_{j} \psi_{i j}-\zeta^{T} \nabla_{i} T-\zeta^{c} \nabla_{i} \mu_{c}-\zeta^{E} E_{i} .
$$

The reversible couplings, eqs. (35)-(42), do not contain contributions $\sim \psi_{i j}$ and are therefore also valid for the case of a permanent network. Thus, in a $\varepsilon \mathrm{T}_{d}$ phase with permanent elasticity there are contributions $\sim T_{i j k}$ to the reversible parts of the stress tensor and the temperature, concentration, and electric currents. The reversible part of the elasticity current is written as

$$
X_{i}^{\varepsilon R}=\Gamma_{4} T_{i j k} A_{j k}
$$

replacing eq. (39).

\section{Appendix A.2. The chiral $\varepsilon \mathrm{T}$ phase in the presence of a permanent network}

Here we focus on the changes compared to the $\varepsilon \mathrm{T}_{d}$ phase brought along by the existence of the pseudoscalar $q_{0}$. For the static considerations nothing changes when compared to the analysis given in sect. 3.2. The same applies to the reversible dynamics, since no $\psi_{i j k}$ contributions are involved and eqs. (59)-(65) remain unchanged. Thus, in a $\varepsilon \mathrm{T}$ phase with permanent elasticity there are chiral contributions $\sim T_{i j k}$ to the reversible parts of the stress tensor and the quasi-currents related to relative rotations and rotations of the octupolar structure.

In the dissipation function, eq. (66), the contributions $\sim \psi_{i j}$ given in the last line have to be zero in the case of a permanent elasticity. As a result there are no specific chiral dissipative effects related to a permanent network. In particular, the chiral part of the elasticity current vanishes, $X_{i}^{\varepsilon D}=0$, replacing eq. (73). In addition, in eqs. (71) and (72) the contributions $\sim \chi^{\Gamma \psi}$ and $\sim \chi^{J \psi}$, respectively, are absent.

Open access funding provided by Max Planck Society (or associated institution if applicable).

Open Access This is an open access article distributed under the terms of the Creative Commons Attribution License (http://creativecommons.org/licenses/by/4.0), which permits unrestricted use, distribution, and reproduction in any medium, provided the original work is properly cited.

\section{References}

1. G. Pelzl, A. Eremin, S. Diele, H. Kresse, W. Weissflog, J. Mater. Chem. 12, 2591 (2002).

2. T. Niori, J. Yamamoto, H. Yokoyama, Mol. Cryst. Liq. Cryst. 409, 475 (2004).

3. Y. Jang, R. Balachandran, C. Keith, A. Lehmann, C. Tschierske, J.K. Vij, Soft Matter 8, 10479 (2012).
4. O.N. Kadkin, E.H. Kim, Y.J. Rha, S.Y. Kim, J. Taem, M.-G. Choi, Chem. Eur. J. 15, 10343 (2009).

5. E.H. Kim, O.N. Kadkin, S.Y. Kim, J. Taem, M.-G. Choi, Eur. J. Inorg. Chem. 2011, 2933 (2011).

6. H.R. Brand, H. Pleiner, P.E. Cladis, Physica A 351, 189 (2005).

7. H.R. Brand, H. Pleiner, Eur. Phys. J. E 31, 37 (2010).

8. H. Pleiner, H.R. Brand, Eur. Phys. J. E 37, 11 (2014).

9. H. Pleiner, H.R. Brand, Braz. J. Phys. 46, 565 (2016).

10. P.G. de Gennes, The Physics of Liquid Crystals (Clarendon Press, Oxford, 1975).

11. S. Chandrasekhar, Liquid Crystals (Cambridge University Press, 1977).

12. P.G. de Gennes, J. Prost, The Physics of Liquid Crystals (Oxford University Press, Oxford, 1995).

13. M. Jasinski, D. Pociecha, H. Monobe, J. Szczytko, P. Kaszynski, J. Am. Chem. Soc. 136, 14658 (2014).

14. C. Dressel, T. Reppe, M. Prehm, M. Brautzsch, C. Tschierske, Nat. Chem. 6, 971 (2014).

15. C. Dressel, W. Weissflog, C. Tschierske, Chem. Commun. 51, 15850 (2015).

16. M. Alaasar, M. Prehm, Y. Cao, F. Liu, C. Tschierske, Angew. Chem. Int. Ed. 55, 312 (2016).

17. C. Tschierske, G. Ungar, ChemPhysChem. 17, 9 (2016).

18. L.G. Fel, Phys. Rev. E 52, 702 (1995).

19. V.P. Panov, M. Nagaraj, J.K. Vij, Y.P. Panarin, A. Kohlmeier, M.G. Tamba, R.A. Lewis, G.H. Mehl, Phys. Rev. Lett. 105, 167801 (2010).

20. R. Balachandran, V.P. Panov, Y.P. Panarin, J.K. Vij, M.G. Tamba, G.H. Mehl, J.K. Song, J. Mater. Chem. C 2, 8179 (2014).

21. A. Hoffmann, A.G. Vanakaras, A. Kohlmeier, G.H. Mehl, J.D. Photinos, Soft Matter 11, 850 (2015).

22. L. Longa, G. Pajak, T. Wydro, Phys. Rev. E 79, 040701 (2009).

23. L.G. Fel, Phys. Rev. E 52, 2692 (1995).

24. L. Radzihovsky, T.C. Lubensky, Europhys. Lett. 54, 206 (2001).

25. L. Radzihovsky, T.C. Lubensky, Phys. Rev. E 66, 031704 (2002).

26. H.R. Brand, H. Pleiner, P.E. Cladis, Eur. Phys. J. E 7, 163 (2002).

27. P.G. de Gennes, Mol. Cryst. Liq. Cryst. 12, 191 (1971).

28. W.P. Mason, Physical Acoustics and the Properties of Solids (D. Van Nostrand, New York, 1958).

29. H.R. Brand, H. Pleiner, W. Renz, J. Phys. (Paris) 51, 1065 (1990).

30. H. Pleiner, H.R. Brand, Mol. Cryst. Liq. Cryst. 199, 407 (1991).

31. H. Temmen, H. Pleiner, M. Liu, H.R. Brand, Phys. Rev. Lett. 84, 3228 (2000).

32. H. Pleiner, M. Liu, H.R. Brand, Rheol. Acta 39, 560 (2000).

33. H. Temmen, H. Pleiner, M. Liu, H.R. Brand, Phys. Rev. Lett. 86, 745 (2001)

34. M. Grmela, Phys. Lett. A 296, 97 (2002).

35. H. Pleiner, M. Liu, H.R. Brand, Rheol. Acta 41, 375 (2002).

36. H. Pleiner, M. Liu, H.R. Brand, Rheol. Acta 43, 502 (2004).

37. H. Pleiner, M. Liu, H.R. Brand, in IMA Volume in Mathematics and its Applications, Vol. 141, edited by M.-C.T. Calderer, E.M. Terentjev (Springer, Berlin, 2005) p. 99. 
38. H.R. Brand, A. Fink, H. Pleiner, Eur. Phys. J. E 38, 65 (2015).

39. O. Müller, M. Liu, H. Pleiner, H.R. Brand, Phys. Rev. E 93, 023113 (2016).

40. O. Müller, M. Liu, H. Pleiner, H.R. Brand, Phys. Rev. E 93, $023114(2016)$

41. N.D. Mermin, T.L. Ho, Phys. Rev. Lett. 36, 594 (1976).

42. H.R. Brand, H. Pleiner, Phys. Rev. A 24, 2777 (1981).

43. M. Liu, Phys. Rev. A 24, 2720 (1981).

44. H.R. Brand, H. Pleiner, Phys. Rev. D 24, 3048 (1981).

45. A.M. Menzel, H. Pleiner, H.R. Brand, J. Chem. Phys. 126, 234901 (2007).

46. P.G. de Gennes, in Liquid Crystals of One- and TwoDimensional Order, edited by W. Helfrich, G. Heppke (Springer, Heidelberg, 1980) p. 231ff.

47. S. Bohlius, H.R. Brand, H. Pleiner, Phys. Rev. E 70, 061411 (2004).

48. H.R. Brand, Makromol. Chem. Rapid Commun. 10, 441 (1989).

49. A.M. Menzel, H.R. Brand, J. Chem. Phys. 125, 194704 (2006).

50. S.R. de Groot, P. Mazur, Nonequilibrium Thermodynamics (North Holland, Amsterdam, 1962).
51. P. Martin, O. Parodi, P.S. Pershan, Phys. Rev. A 6, 2401 (1972).

52. D. Forster, Hydrodynamic Fluctuations, Broken Symmetry and Correlation Functions (Benjamin, Reading, Mass., 1975).

53. H. Pleiner, H.R. Brand, in Pattern Formation in Liquid Crystals, edited by A. Buka, L. Kramer (Springer, New York, 1996) p. 15.

54. H.R. Brand, H. Pleiner, Physica A 208, 359 (1994).

55. H. Pleiner, H.R. Brand, EPL 89, 26003 (2010).

56. H.R. Brand, H. Pleiner, Phys. Rev. A 37, 2736 (1988).

57. H.R. Brand, H. Pleiner, Encyc. Mater.: Sci. Technol. 5, 1214 (2001)

58. H.R. Brand, H. Pleiner, Cholesteric Liquid Crystals: Flow Properties, Thermo- and Electromechanical Coupling, in Reference Module in Materials Science and Materials Engineering, edited by Saleem Hashmi (Elsevier, Oxford, 2016) pp. 1-8.

59. D. Svenšek, H. Pleiner, H.R. Brand, Phys. Rev. E 78, 021703 (2008).

60. H.R. Brand, H. Pleiner, D. Svenšek, Phys. Rev. E 88, 024501 (2013). 